This is the accepted version of the following article:

Quesada-González D., Stefani C., González I., de la Escosura-Muñiz A., Domingo N., Mutjé P., Merkoçi A.. Signal enhancement on gold nanoparticle-based lateral flow tests using cellulose nanofibers. Biosensors and Bioelectronics, (2019). 141. 111407: - . 10.1016/j.bios.2019.111407,

which has been published in final form at https://dx.doi.org/10.1016/j.bios.2019.111407 (c) https://dx.doi.org/10.1016/j.bios.2019.111407. This manuscript version is made available under the CC-BY-NC-ND 4.0 license http://creativecommons.org/licenses/by-nc-nd/4.0/ 


\title{
Signal enhancement on gold nanoparticle-based lateral flow tests using cellulose nanofibers
}

\author{
Daniel Quesada-González ${ }^{\mathrm{a}}$, Christina Stefani ${ }^{\mathrm{b}}$, Israel González ${ }^{\mathrm{c}}$, Alfredo de la Escosura-Muñiz ${ }^{\mathrm{a}}$, Neus \\ Domingo $^{\mathrm{b}}$, Pere Mutjéc ${ }^{\mathrm{c}}$, Arben Merkoçi ${ }^{\mathrm{a}}{ }^{\mathrm{d} *}$
}

\author{
${ }^{a}$ Nanobioelectronics \& Biosensors Group, Catalan Institute of Nanoscience and Nanotechnology (ICN2), CSIC and BIST, Campus UAB, Bellaterra, 08193 \\ Barcelona, Spain. \\ ${ }^{b}$ Advanced Atomic Force Microscopy Laboratory, Catalan Institute of Nanoscience and Nanotechnology (ICN2), CSIC and BIST, Campus UAB, Bellaterra, \\ 08193 Barcelona, Spain. \\ ${ }^{c}$ LEPAMAP Research group, University of Girona, C/Maria Aurèlia Capmany, 61, 17071 Girona, Spain. \\ 'ICREA, Institució Catalana de Recerca i Estudis Avançats, Pg. Lluís Companys 23, 08010 Barcelona, Spain. \\ *arben.merkoci@icn2.cat
}

\begin{abstract}
Lateral flow paper-based biosensors merge as powerful tools in point-of-care diagnostics since they are cheap, portable, robust, selective, fast and easy to use. However, the sensitivity of this type of biosensors is not always as high as required, often not permitting a clear quantification. To improve the colorimetric response of standard lateral flow strips (LFs), we have applied a new enhancement strategy that increases the sensitivity of LFs based on the use of cellulose nanofibers (CNF). CNF penetrate inside the pores of LFs nitrocellulose paper, compacting the pore size only in the test line, particularly near the surface of the strip. This modification retains the bioreceptors (antibodies) close to the surface of the strips, and thus further increasing the density of selectively attached gold nanoparticles (AuNPs) in the top part of the membrane, in the test line area, only when the sample is positive. This effect boosts in average a $36.6 \%$ the sensitivity of the LFs. The optical measurements of the LFs were carried out with a mobile phone camera whose imaging resolution was improved by attaching microscopic lens on the camera objective. The characterization of CNF into paper and their effect was analyzed using atomic force microscope (AFM) and scanning electron microscope (SEM) imaging techniques.
\end{abstract}

\section{Introduction}

The World Health Organization (WHO) urges the development of affordable, sensitive, selective, user-friendly, rapid and robust, equipment-free and derivable to the end-user devices (ASSURED criteria) (LaBarre et al., 2011). Lateral flow strips (LFs) are paper-based biosensors that emerge as powerful devices since they fulfill all the demands (Wong and Tse, 2009; Parolo and Merkoçi, 2013; Quesada-González and Merkoçi, 2015; López-Marzo and Merkoçi, 2016; Zhan et al., 2017; Quesada-González, 2018). Paper substrate is inexpensive, recyclable, tunable and allows the storage of bioreceptors (e.g. antibodies [Wu et al., 2014]) and nanomaterials (QuesadaGonzález and Merkoçi, 2015; Zhan et al., 2017) in dry state. These capabilities make LFs suitable for point-of-care (PoC) diagnostics (Burns, 2002; Kratz and Lewandrowski, 2003; Quesada-González and Merkoçi, 2018). However, in some cases LFs may present not enough sensitivity levels, which does not allow or makes it difficult to perform quantitative analysis. Therefore, many studies and signal enhancement strategies have been proposed during last years, as they are: the addition of extra amplification steps (Fu et al., 2010, 2011; Fridley et al., 2012; Rodriguez et al., 2016), secondary chemical reactions (Anfossi et al., 2013; Parolo et al., 2013a; Chapman et al., 2015; Loynachan et al., 2018), architecture modifications (Parolo et al., 2013b; Rivas et al., 2014), new labels (Quesada-González et al., 2019) or even exploring alternative signal translation methods beyond the optical response (Tang et al., 2009; Marquina et al., 2012; Li et al., 2014; Zhu et al. 2014; Khlebtsov et al., 2019).

The porosity of paper is a key factor regarding the sensitivity of the strips (Wong and Tse, 2009). Small pores will lead to higher sensitivity, as we demonstrated in a previous work (Rivas et al., 2014), nevertheless the sample may have difficulties to flow, increasing the assay time and the probability of having a flow stop or membrane defects, further provoking low reproducibility. Herein, we propose to decrease the pore size of the membrane only on the test area, where the recognition antibodies are dispensed. To accomplish this milestone, in this work we have included for the first time cellulose nanofibers $(\mathrm{CNF})$ in the test area. CNF are a nanomaterial often used as reinforcement additive on papermaker pulps (González et al., 2012, 2013, 2014, 2017; Alcalá et al. 2013; Delgado-Aguilar et al., 2015, 2016; Tarrés et al., 2016). This nanomaterial can be produced as a gel that is easily dispensed and dried inside the paper pores. In addition to the structural modification, $\mathrm{CNF}$ are biocompatible with antibodies, thus increasing the areas where they can be attached, so that more of them are retained near the paper surface, where it is best appreciated the color of transducer particles (Fig. 1A). Gold nanoparticles (AuNPs) with average diameter of $18.0 \pm 0.5 \mathrm{~nm}$ (Fig. S1) have been chosen as transducer particles since they are easy to synthetize (Turkevich et al., 1951), bioconjugate (Shyu et al., 2001; De La EscosuraMuñiz et al., 2010; Parolo et al., 2013c; Rivas et al., 2015; Ngu et al., 2019) and have a strong red color readily detectable (Fu et al., 2016; Quesada-González et al., 2018). Furthermore, AuNPs have demonstrated their applicability in the evaluation of real samples with LFs (Shim et al., 2009; Xie et al., 2015; Quesada-González et al., 2018; Liu et al., 2018). Our LFs design is represented in Fig. 1B.

The functionality of our LFs devices is finally strongly dependent on the color contrast detection of the test stripe lines. In this sense, mobile phones become important support tools in nowadays' biosensing (Mudanyali et al., 2012; Coskun et al., 2013a, 2013b; Roda et al., 2014, 2016; Berg et al., 2015; Quesada-González and Merkoçi, 2016; Álvarez-Diduk et al., 2017; Kühnemund et al., 2017; You et al., 2017; Brangel et al. 2018), not only due the capacity to record and store the data, but also because of the portability, cloud storage, accessibility and software capabilities that these devices offer. In this work we attach microscopic lenses (Blips Microlenses [Smart Micro Lenses, online reference]) to the mobile phone camera improving the quality of close-up images taken of the test area in LFs, therefore increasing the number of pixels that can be read and 
demonstrating the operability of the device in conjugation with standard camera-based detection.

\section{Experimental section}

\subsection{Materials.}

Tetrachloroauric acid $\left(\mathrm{HAuCl}_{4}\right)$, bovine serum albumin (BSA), goat anti-human IgG antibody, human IgG, trisodium citrate, sucrose, Tween 20, phosphate buffer saline (PBS) tablets and the reagents to prepare phosphate buffer non-saline (PB; sodium phosphate basic and dibasic) and borate buffer (BB; sodium tetraborate and boric acid) were purchased from Sigma Aldrich. Chicken anti-goat antibody was purchased from Abcam.

Nitrocellulose membrane (HF180), cotton membrane (CFSP001700), glass fiber (GFCP00080000) and supporting adhesive cards were purchased from Millipore.

CNF $(0.92 \%)$ were provided by LEPAMAP group, fabricated following a reported protocol (Delgado-Aguilar et al., 2015) by using commercial bleached pine tree pulp as raw material, provided by Ence: Celulosas y Energía S.A.

Blips Lens Basic Kit, from Smart Micro Optics (Smart Micro Lenses, online reference), was purchased in a Kickstarter campaign.

\subsection{Synthesis of gold nanoparticles.}

Following Turkevich method (Turkevich et al., 1951), AuNPs of approximately $20 \mathrm{~nm}$ diameter were synthesized by citrate reduction of $\mathrm{HAuCl}_{4} .50 \mathrm{~mL}$ of a $0.01 \%(\mathrm{w} / \mathrm{v})$ solution of $\mathrm{HAuCl}_{4}$ were prepared in Milli-Q water. This solution was taken to boiling point and $1.25 \mathrm{~mL}$ of $1 \%(\mathrm{w} / \mathrm{v})$ sodium citrate solution was added under continuous vigorous stirring. The solution was kept boiling for 10 more minutes and then allowed to cool down to room temperature. The AuNP solution was adjusted to a $\mathrm{pH}$ of 9.0 by addition of $0.01 \mathrm{mM} \mathrm{BB}$. AuNPs were characterized by transmission electron microscope (TEM, Tecnai F20) as shown in Fig. S1.

\subsection{Gold nanoparticles conjugation.}

$100 \mu \mathrm{L}$ of goat anti-human IgG antibody $(100 \mu \mathrm{g} / \mathrm{mL}$ in $\mathrm{PB})$ were added to $1.5 \mathrm{~mL}$ of AuNP solution and incubated for 20 minutes at room temperature. BSA solution $(0.1 \%(\mathrm{w} / \mathrm{v}), 100$ $\mu \mathrm{L}$ ) was then added to the mixture, which was incubated for another 20 minutes and then centrifuged at $4^{\circ} \mathrm{C}(18000 \mathrm{rcf}, 20$ minutes). The supernatant was discarded and the pellet was reconstituted in $0.5 \mathrm{~mL} \mathrm{BB}(2 \mathrm{mM}, \mathrm{pH} 7.4)$ containing $10 \%$ sucrose.

\subsection{Lateral flow strips preparation.}

The conjugate pad was prepared by dipping the glass fiber into the AuNP-antibody conjugate suspension. The fiber was dried in a vacuum chamber for 3-4 hours, and then stored in barrier zip-lock pouches with drying pearls at $4{ }^{\circ} \mathrm{C}$. The sample pad was prepared by dipping the cotton membrane in PBS buffer containing $0.05 \%$ Tween 20 and $5 \%$ BSA. The pad was dried in the oven at $60^{\circ} \mathrm{C}$ for 2 hours and stored in barrier zip-lock pouches with drying pearls at $4{ }^{\circ} \mathrm{C}$. The detection pad was made assembling nitrocellulose membrane onto a supporting adhesive card.

CNF gel $(0.92 \%)$ was diluted by half (to $0.46 \% \mathrm{CNF}$ ) with Milli $\mathrm{Q}$ water to make it less viscous and able to be dispensed using a Biofluidix Biospot Workstation. It was dispensed over nitrocellulose membrane forming a line. The line was dispensed repeatedly on the same position to increase the concentration of
CNF within nitrocellulose pores (from one to six times; i.e. 0.46 to $2.76 \%$, respectively). After drying the membrane overnight at room temperature, goat anti-human $\mathrm{IgG}$ antibody $(1.0 \mathrm{mg} / \mathrm{mL}$ in PB) was dispensed over the previous line, forming the test line (TL). Chicken anti-goat antibody $(1.0 \mathrm{mg} / \mathrm{mL}$ in $\mathrm{PB})$ was dispensed at $5.5 \mathrm{~mm}$ of distance from the TL (Fig. 2), forming the control line (CL). The detection pad was dried at $37^{\circ} \mathrm{C}$ for 2 hours and stored at room temperature under dry conditions.

As represented on Fig. 2, the conjugate pad was assembled onto the adhesive card with a $1 \mathrm{~mm}$ overlap over the detection pad, on the farthest side from the detection lines. The sample pad was assembled overlapping the conjugate pad at the end of the card. An absorption pad (untreated cellulose membrane) was assembled on the other end of the card, overlapping the detection pad, at $4 \mathrm{~mm}$ away from the CL. The strips were cut to a width of $6 \mathrm{~mm}$ using a guillotine and stored at room temperature with drying pearls.

\subsection{Assay performance.}

Human IgG solutions, from 0.01 to $1.00 \mu \mathrm{g} / \mathrm{mL}$, were prepared in $\mathrm{pH} 7.4 \mathrm{~PB}$ (buffer that was also used as blank sample). $200 \mu \mathrm{L}$ of each concentration were dropped on the sample pad of the strips. After 5 minutes, TL and CL were observed. Images of the strips were taken at $6 \mathrm{~mm}$ of distance using a Moto $\mathrm{Z}$ mobile phone camera (13 megapixel) in which Micro Lens, from Blips Lens Basic Kit, were sticked on the objective (Fig. S2). A 3D-printed tool was made using BCN-3D Sigma printer (melting polylactic acid filament) in order to keep the distance between the strips and the camera constant (video V1).

Image J software (Álvarez-Diduk et al., 2017; Kühnemund et al., 2017; Quesada-González et al., 2018) was used to quantify the TL intensity of color in the images. The intensity of white pixels (maximum value 255 , being 0 the blackest color) was measured in 8-bit black-and-white format reconverted images (Fig. S3 illustrates the software and the area which was selected in a TL). Data was normalized into \% color where $0 \%$ and 100 $\%$ equal to 255 and 0 values respectively by the equation:

\section{$\%$ color $=100-(100 \cdot$ IMGJ $) / 255$}

Where "IMGJ" is the value measured with the software (from 0 to 255$)$.

\subsection{Characterization of CNF on nitrocellulose.}

After the assay, two positive LFs were selected and the areas corresponding to the TL were cut for its study. The sample concentration added in both strips was the same, being one strip made with CNF gel on the TL and the other without. Both samples were observed on scanning electron microscopy (SEM; FEI Magellan 400L XHR).

The same samples were observed in atomic force microscopy (AFM). AFM imaging was performed with a MFP-3D Asylum AFM (Oxford Instruments, Scotts Valley, CA). In all the experiments, PPP-EFM tips (Nanosensors; Schaffhausen, Switzerland) with a stiffness constant $\mathrm{k}=2 \mathrm{~N} / \mathrm{m}$ and coated with Ptlr5 were used. Multi-Frequency AFM is based in the use of multiple excitation modes with different characteristic frequencies. In this case, the fundamental excitation mode was used together with the second eigenmode.

In simple bimodal AFM operation mode, the tip is excited at both frequencies, with amplitudes A2 < A1. A remarkable benefit of MF bimodal imaging is the fact that the forces between the tip and the sample are very small while still allowing a clear non- 
destructive differentiation of composition, which makes it a very convenient way to investigate samples composed of different materials. The excitation at the fundamental mode was used to measure the topography in amplitude-modulation AFM, while changes in amplitude and phase for the second eigenmode, not affected by the feedback loop restrictions, are monitored. The contrast obtained for the second eigenmode arises mainly on the different composition of the sample and change of mechanical properties such as stiffness of the material. Moreover, this technique is depth sensitive and allows to observe the presence of structures embedded in a matrix such as a gel, even below the surface.

It must be highlighted that, in a previous work (Tarrés et al., 2017), CNF were characterized by XRD exhibiting the typical Cellulose I crystalline structure, with peaks corresponding to (110), (110) and (200) reflection planes and an average diameter around $20 \mathrm{~nm}$.

\section{Results and discussion}

AuNPs-based LFs were prepared for the detection of human immunoglobulin ( $\mathrm{HIgG}$ ) (as described in methods section), a protein often used as model on the optimization of lateral flow assays (Parolo et al., 2013a, 2013b; Rivas et al., 2014; QuesadaGonzález et al., 2019). CNF gel was dispensed on the TL of LFs, before adding the antibodies, to increase the density of these retained near the surface of the strip and thus, enhance the colorimetric response observable. Atomic force microscopy (AFM) images shown in Fig. 3 demonstrate the presence of CNF on nitrocellulose samples.

To further analyze the role of the CNF on the enhancement of AuNPs binding, the same samples were also observed by AFM. Initially, AFM was tested as a useful tool to characterize the presence of CNFs. A sample of CNF gel was studied by bimodal AFM and the presence of CNF was clearly observed due to mechanical stiffness contrast with respect to the medium (Fig. S4). They show an average diameter of $\sim 20 \mathrm{~nm}$ (it is to mention that due to the density of the sample, the fibers are entangled and it is not possible to have them isolated). The diameter fits with the results previously observed (Tarrés et al., 2017), in which the $\mathrm{CNF}$ were characterized by XRD. To further prove the existence of CNF on nitrocellulose membranes, two different nitrocellulose samples with and without CNF were analyzed by bimodal AFM, as shown in Fig. 3. An area of $1 \mu \mathrm{m}$ of the surface of a nitrocellulose membrane was scanned - maximum possible as limited by the degree of curvature of these surfaces due to the mean radius of the nitrocellulose fiber. The topography image shown in Fig 3a gives evidence of the intrinsic rugosity of the nitrocellulose membrane. Still, these features do not induce significant contrast in the bimodal AFM images shown in Fig. 3b and $3 \mathrm{c}$, indicating the homogeneity of the material. Instead, Fig $3 \mathrm{~d}$ clearly demonstrates the presence of CNF deposited on the nitrocellulose membrane as evidenced by both, the features in the topography and their further contrast in bimodal AFM images (Garcia and Proksch, 2013; González-Domínguez et al., 2016). In this case, the CNF seem to be retained mainly in one side of the fiber, the one where TL antibodies are dispensed, that which is visible at bare eye.

To demonstrate that CNF gel can increase the density of antibodies near the paper surface, thus enhancing the signal (i.e. quantity of AuNPs retained on positive samples), four concentrations of this nanomaterial were dispensed on nitrocellulose. Different concentrations of $\mathrm{HIgG}$ were dropped on the distinct LFs, without CNF and with the different four CNF concentrations, and the intensities were recorded by a mobile phone camera with magnification lens attached on the objective. Results obtained are shown on Fig. 4. It can be observed how, the higher the concentration of CNF within nitrocellulose, the higher is the color enhancement of TL up to a CNF concentration of $1.84 \%$. At higher concentrations of the gel the intensity stops being enhanced. Seemingly an excess of CNF impedes AuNPs to attach on nitrocellulose pores. Thus, $1.84 \%$ of CNF was chosen as optimal for the signal enhancement of LFs and applied on the further experiments. Also, it is observed how the sensitivity seems to increase especially at lower concentrations; because of that, on the following experiments more concentrations between $0-0.5 \mu \mathrm{g} / \mathrm{mL}$ were evaluated.

Standard LFs versus CNF-LFs (containing $1.84 \% \mathrm{CNF}$ in the TL) were compared. Different concentrations of $\mathrm{HIgG}$ were added on the strips and the intensities were measured as explained at methods section. As shown in Fig. 5, over a concentration of $0.05 \mu \mathrm{g} / \mathrm{mL} \mathrm{HIgG}$ the signal of the CNF-LFs is increased by an average of $36.6 \%$ in comparison to those strips without CNF gel. In absence of HIgG (i.e. blank samples), the response of CNF-LFs and standard LFs is comparable. It demonstrates that there is no unspecific adsorption between HIgG and the CNFs. So that, AuNPs only stop on TL due the interaction of antibodies and the analyte.

The calibration curve (Fig. S5) obtained for CNF-LFs follows the equation:

\section{$\%$ color $=5.688 \ln [\mathrm{HIgG}(\boldsymbol{\mu g} / \mathrm{mL})]+57.768$}

The $r$ value for the equation was 0.995 . The limit of detection (LOD) was calculated as $\%$ color $_{\text {LOD }}=$ blank $+3 \sigma_{\text {blank }}$, i.e., the corresponding value of blank sample plus 3 times its standard deviation (Armbruster, 2008). The value obtained was of 0.01 $\mu \mathrm{g} / \mathrm{mL}$ in both cases. Even though the LOD is similar to standard LFs (since it only depends on the antibodies affinity for the $\mathrm{HIgG}$, not on the CNF), the sensitivity is highly improved with the CNF (the slope was almost two-times higher than the slope of standard LFS, $5.7 \pm 0.1$ vs. $3.2 \pm 0.1)$. The method shows a reproducibility (relative standard deviation, RSD) of $2 \%(n=6)$ for a $\mathrm{HIgG}$ concentration of $0.05 \mu \mathrm{g} / \mathrm{mL}$.

These results reflect what was observed when characterizing the strips by SEM and AFM (Fig 6). In SEM images it can be observed how, for the same amount of HIgG, the population of AuNPs on the TL is greater on the CNF-LFs. It demonstrates that more AuNPs are being retained, at least on the surface of the strip. Still, SEM does not allow the imaging of CNFs due to lack of SEM contrast against the CNFs.

Finally, the binding of AuNP on the TL was also studied by AFM. It was demonstrated that for a NFC-LFs the quantity of AuNPs is significantly greater than the case of the LFs without CNF (Fig. 6). The high roughness level of the curved surfaces impeded to selectively analyze the binding mechanisms between single AuNP and the CNF, but it was possible to determine that the higher density of AuNP were placed on the side areas of nitrocellulose for which also higher density of CNFs were observed to deposit, for the CNF functionalized strips, while for the non-functionalized ones, the attachment of AuNPs seemed to be randomly dispersed on the nitrocellulose surface.

\section{Conclusions}

We have devised a new signal enhancement strategy for LFs consisting in the addition of CNF on paper nitrocellulose to decrease the pore size in the TL, thus increasing the amount of bioreceptors (capture antibodies) near the surface. Thanks to this modification, on positive samples we observed an average 
increase of $36.6 \%$ of the colorimetric signal, which means that effectively we succeed increasing the amount of AuNPs retained. This allows to increase the sensitivity of the LF immunoassay (HIgG evaluated as model analyte) as well as to better discriminate between different analyte concentrations, which is of great relevance for both naked-eye and instrumental-based detection. Unspecific adsorption of AuNPs on TL is not observed (when measuring blank HIgG samples on a CNF-LFs), which avoids false positive results. Due to these conclusions, we believe that our strategy can be used to discern especially at low concentrations, which would be useful especially on diagnostic applications. The characterization of the nitrocellulose performed with SEM and AFM demonstrates the presence of the CNF and how it affects to the interaction with AuNPs. The proposed modification is simple and cheap and can be easily applied to any type of LF strip, enabling its use in PoC applications.

\section{Acknowledgments}

The ICN2 is funded by the CERCA Programme / Generalitat de Catalunya. The ICN2 is supported by the Severo Ochoa program of the Spanish Ministry of Economy, Industry and Competitiveness (MINECO, grant No. SEV-2017-0706). Financial support was obtained under MAT2017-87202-P, funded by Agencia Estatal de Investigación (AEI) and Fondo Europeo de Desarrollo Regional (FEDER), and MINECO project FIS2015-73932-JIN. This work was partially funded by 2017SGR-579 project from the Generalitat de Catalunya. D.Q.G. acknowledges Universitat Autònoma de Barcelona for the possibility of carrying out this work inside the framework of its $\mathrm{PhD}$ Programme in Chemistry. Ch.S. thanks the BIST for a preBIST Grant (No. 754558). We thank Marcos Rosado and Belén Ballesteros for the support given on SEM and TEM imaging. The music used on video V1 is "Converging Lines" by David Hilowitz, used under the Creative Commons Attribution Non-Commercial 4.0 International License (CC BY-NC 4.0), https://bit.ly/2LxhpAJ.

\section{References}

Alcalá, M.; González, I.; Boufi, S.; Vilaseca, F.; Mutjé, P. Cellulose 2013, 20, 2909-2921.

Álvarez-Diduk, R.; Orozco, J.; Merkoçi, A. Sci. Rep. 2017, 7, 976. Anfossi, L.; Di Nardo, F.; Giovannoli, C.; Passini, C.; Baggiani, C. Anal. Bioanal. Chem. 2013, 405, 9859-9867.

Armbruster, D. A.; Pry, T. Clin. Biochem. Rev. 2008, 29, 49-52. Berg, B.; Cortazar, B.; Tseng, D.; Ozkan, H.; Feng, S.; Wei, Q.; Chan, R. Y. L.; Burbano, J.; Farooqui, Q.; Lewinski, M.; Carlo, D.; Garner, O. B.; Ozcan, A. ACS Nano 2015, 9, 7857-7866.

Brangel, P.; Sobarzo, A.; Parolo, C.; Miller, B. S.; Howes, P. D.; Gelkop, S.; Lutwama, J. J.; Dye, J. M.; McKendry, R. A.; Lobel, L.; Stevens, M. M. ACS Nano 2018, 12, 63-73.

Burns, M. A. Science. 2002, 296, 1818-1819.

Chapman, R.; Lin, Y.; Burnapp, M.; Bentham, A.; Hillier, D.;

Zabron, A.; Khan, S.; Tyreman, M.; Stevens, M. M. ACS Nano 2015, 9, 2565-2573.

Coskun, A. F.; Wong, J.; Khodadadi, D.; Nagi, R.; Tey, A.; Ozcan, A. Lab Chip 2013a, 13, 636-640.

Coskun, A. F.; Nagi, R.; Sadeghi, K.; Phillips, S.; Ozcan, A. Lab Chip 2013b, 13, 4231-4238.

De La Escosura-Muñiz, A.; Parolo, C.; Merkoi, A. Mater. Today 2010, 13, 24-34.

Delgado-Aguilar, M.; González, I.; Tarrés, Q.; Alcalà, M.; Pèlach, M. À.; Mutjé, P. BioResources 2015, 10, 5330-5344.

Delgado-Aguilar, M.; González, I.; Tarrés, Q.; Pèlach, M. À.; Alcalà, M.; Mutjé, P. Ind. Crops Prod. 2016, 86, 295-300.
Fridley, G. E.; Le, H. Q.; Fu, E.; Yager, P. Lab Chip 2012, 12, 43214327.

Fu, E.; Lutz, B.; Kauffman, P.; Yager, P. Lab Chip 2010, 10, 918.

Fu, E.; Ramsey, S. A.; Kauffman, P.; Lutz, B.; Yager, P. Microfluid. Nanofluidics 2011, 10, 29-35.

Fu, Q.; Wu, Z.; Xu, F.; Li, X.; Yao, C.; Xu, M.; Sheng, L.; Yu, S.; Tang, Y. Lab Chip 2016, 16, 1927-1933.

Garcia, R.; Proksch R. Eur. Polym. J. 2013, 49, 1897-1906.

González, I.; Boufi, S.; Pèlach, M. A.; Alcalà, M.; Vilaseca, F.; Mutjé, P. BioResources 2012, 7, 5167-5180.

González, I.; Vilaseca, F.; Alcalá, M.; Pèlach, M. A.; Boufi, S.; Mutjé, P. Cellulose 2013, 20, 1425-1435.

González, I.; Alcalà, M.; Chinga-Carrasco, G.; Vilaseca, F.; Boufi, S.; Mutjé, P. Cellulose 2014, 21, 2599-2609.

González, I.; Oliver-Ortega, H.; Tarrés, Q.; Delgado-Aguilar, M.; Mutjé, P.; Andreu, D. Int. J. Biol. Macromol. 2017, 105, 741-748. González-Domínguez, I.; Gutiérrez-Granados, S.; Cervera, L.; Gòdia, F.; Domingo, N. Biophysical Journal, 2016, 111, 1173-1179. Khlebtsov, B. N.; Bratashov, D. N.; Byzova, N. A.; Dzantiev, B. B.; Khlebtsov, N. G. Nanoresearch 2019, 12(2), 413-420.

Kratz, A.; Lewandrowski, K. B. Arch. Pathol. Lab. Med. 2003, 127, 511.

Kühnemund, M.; Wei, Q.; Darai, E.; Wang, Y.; Iván, H. N.; Yang, Z.; Tseng, D.; Ahlford, A.; Mathot, L.; Sjöblom, T.; Aydogan, O.; Nilsson, M. Nat. Commun. 2017, 8, 1-8.

LaBarre, P.; Boyle, D.; Hawkins, K.; Weigl, B. Int. Soc. Opt. Eng. 2011, 802902 .

Li, M.; Yang, H.; Li, S.; Zhao, K.; Li, J.; Jiang, D.; Sun, L.; Deng, A. J. Agric. Food Chem. 2014, 62, 10896-10902.

Liu, J.; Hu, X.; Cao, F.; Zhang, Y.; Lu, J.; Zeng, L. R. Soc. open sci. 2018, 5, 180288 .

López-Marzo, A. M.; Merkoçi, A. Lab Chip 2016, 16, 3150-3176. Loynachan, C. N.; Thomas, M. R.; Gray, E. R.; Richards, D. A.; Kim, J.; Miller, B. S.; Brookes, J. C.; Agarwal, S.; Chudasama, V.; McKendry, R. A.; Stevens, M. M. ACS Nano 2018, 12, 279-288. Marquina, C.; De Teresa, J. M.; Serrate, D.; Marzo, J.; Cardoso, F. A.; Saurel, D.; Cardoso, S.; Freitas, P. P.; Ibarra, M. R. J. Magn. Magn. Mater. 2012, 324, 3495-3498.

Mudanyali, O.; Dimitrov, S.; Sikora, U.; Padmanabhan, S.; Navruz, I.; Ozcan, A. Lab Chip 2012, 12, 2678-2686.

Ngu, M. A. V.; Bergantin, J. H.; Ramos, J. D. A. Protein Peptide Lett. 2019, 26, doi: 10.2174/0929866526666190212164751. Parolo, C.; Merkoçi, A. Chem. Soc. Rev. 2013, 42, 450-457. Parolo, C.; de la Escosura-Muñiz, A.; Merkoçi, A. Biosens. Bioelectron. 2013a, 40, 412-416.

Parolo, C.; Medina-Sánchez, M.; de la Escosura-Muñiz, A.; Merkoçi, A. Lab Chip 2013b, 13, 386-390.

Parolo, C.; De La Escosura-Muñiz, A.; Polo, E.; Grazú, V.; De La Fuente, J. M.; Merkoçi, A. ACS Appl. Mater. Interfaces 2013c, 5, 10753-10759.

Quesada-González, D.; Merkoçi, A. Biosens. Bioelectron. 2015, 73, $47-63$.

Quesada-González, D.; Merkoçi, A. Biosens. Bioelectron. 2016, 92, 549-562.

Quesada-González, D. "Design and application of nanomaterialbased lateral flow devices", Ph.D. Dissertation, Autonomous University of Barcelona, Bellaterra, Spain, 2018

Quesada-González, D.; Merkoçi, A. Chem. Soc. Rev. 2018, 47, 4697-4709.

Quesada-González, D.; Jairo, G. A.; Blake II, R. C.; Blake, D. A.; Merkoçi, A. Sci. Rep. 2018, 8, 16157.

Quesada-González, D.; Sena-Torralba, A.; Wicaksono, W. P.; de la Escosura-Muñiz, A.; Ivandini, T. A.; Merkoçi, A. Biosens. Bioelectron. 2019, 132, 132-135.

Rivas, L.; Medina-Sánchez, M.; de la Escosura-Muñiz, A.; Merkoçi, A. Lab Chip 2014, 14, 4406-4414.

Rivas, L.; de la Escosura-Muñiz, A.; Serrano, L.; Altet, L.; Francino, O.; Sánchez; Merkoçi, A. Nanoresearch 2015, 8(11), 3704-3714. Rodriguez, N. M.; Wong, W. S.; Liu, L.; Dewar, R.; Klapperich, C. M. Lab Chip 2016, 16, 753-763. 
Roda, A.; Michelini, E.; Cevenini, L.; Calabria, D.; Calabretta, M. M.; Simoni, P. Anal. Chem. 2014, 86, 7299-7304.

Roda, A.; Michelini, E.; Zangheri, M.; Di Fusco, M.; Calabria, D.; Simoni, P. TrAC - Trends Anal. Chem. 2016, 79, 317-325.

Shim, W. B.; Kim, K. Y.; Chung, D. H. J. Agric. Food Chem. 2009, 57(10), 4035-4041.

Shyu, R. H.; Shyu, H. F.; Liu, H. W.; Tang, S. S. Toxicon 2001, 40, 255-258.

Smart Micro Optics: Blips lenses [online reference]

https://www.smartmicrooptics.com/blips/ (accessed Mar 16, 2019).

Tang, D.; Sauceda, J. C.; Lin, Z.; Ott, S.; Basova, E.; Goryacheva, I.; Biselli, S.; Lin, J.; Niessner, R.; Knopp, D. Biosens. Bioelectron. 2009, 25, 514-518.

Tarrés, Q.; Delgado-Aguilar, M.; Pèlach, M. A.; González, I.; Boufi, S.; Mutjé, P. Cellulose 2016, 23, 3939-3950.

Tarrés. Q.; Boufi, S; Mutjé, P.; Delgado-Aguilar, M. Cellulose 2017, 24, 3943-3954.

Turkevich, J.; Stevenson, P. C.; Hillier, J. A Faraday Soc. 1951, 11, $55-75$.

Wong, R.; Tse, H. "Lateral Flow Immunoassay", Springer 2009

Wu, G.; Srivastava, J.; Zaman, M. H. Anal. Biochem. 2014, 449 , 147-154.

Xie, Y.; Chang, H.; Zhao, K.; Li, J.; Yang, H.; Mei, L.; Xua, S.; Deng, A. Anal. Methods, 2015, 7, 513-520.

You, M.; Lin, M.; Gong, Y.; Wang, S.; Li, A.; Ji, L.; Zhao, H.; Ling, K.; Wen, T.; Huang, Y.; Gao, D.; Ma, Q.; Wang, T.; Ma, A.; Li, X.; $\mathrm{Xu}, \mathrm{F}$. ACS Nano 2017, 11, 6261-6270.

Zhan, L.; Guo, S. Z.; Song, F.; Gong, Y.; Xu, F.; Boulware, D. R.; McAlpine, M. C.; Chan, W. C. W.; Bischof, J. C. Nano Lett. 2017 17(12), 7207-7212.

Zhu, X.; Shah, P.; Stoff, S.; Liu, H.; Li, C. A Analyst 2014, 139,

2850-2857.

\section{Supplementary Material}

Supplementary material (TEM characterization of AuNPs; Image J software screenshot; AFM imaging of CNF; calibration curves; a video of a 3D printed tool used to control the distance between mobile phone camera-BLIPS Lens and the LFs) is available in the online version of this article at http://xxxxxxxxxxxxxxx. 
Figures

A.
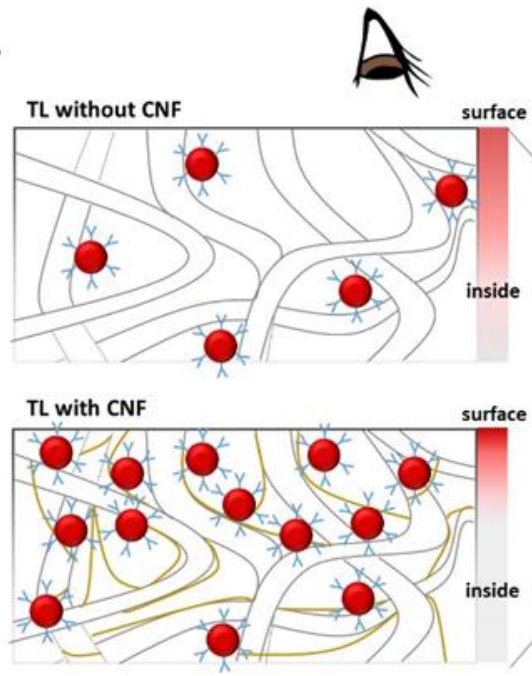

B.

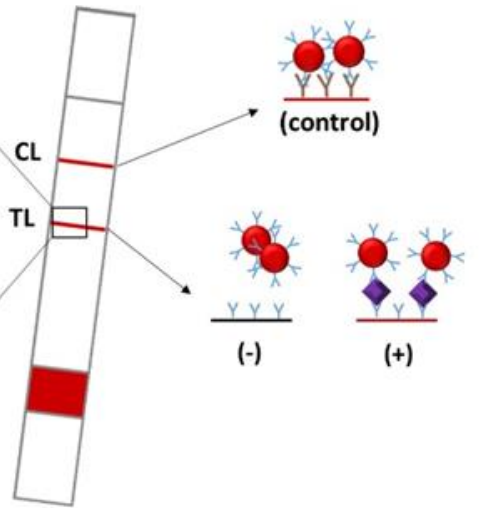

nitrocellulose

cellulose nanofibers (CNF)

AuNPs

Human IgG

Y recognition ab.

Y control ab.

Figure 1. A) Schematic representation of a positive TL not modified (up) and modified with cellulose nanofibers dispensed (down). B) Schematic representation of a LFs with all its components.

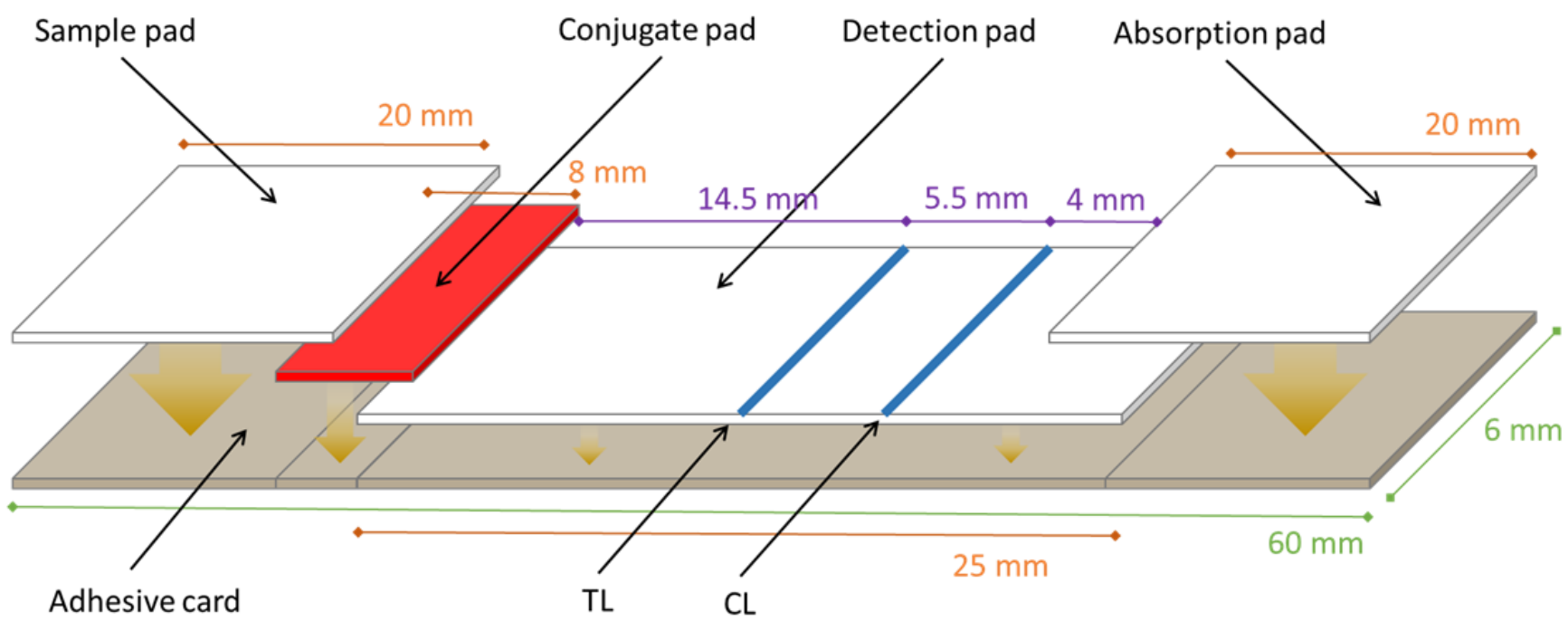

Figure 2. Representation of a LFs construction; the different pads that compose it and its position (in green, size of the adhesive card; in orange, size of the pads; in purple, some positioning parameters). 

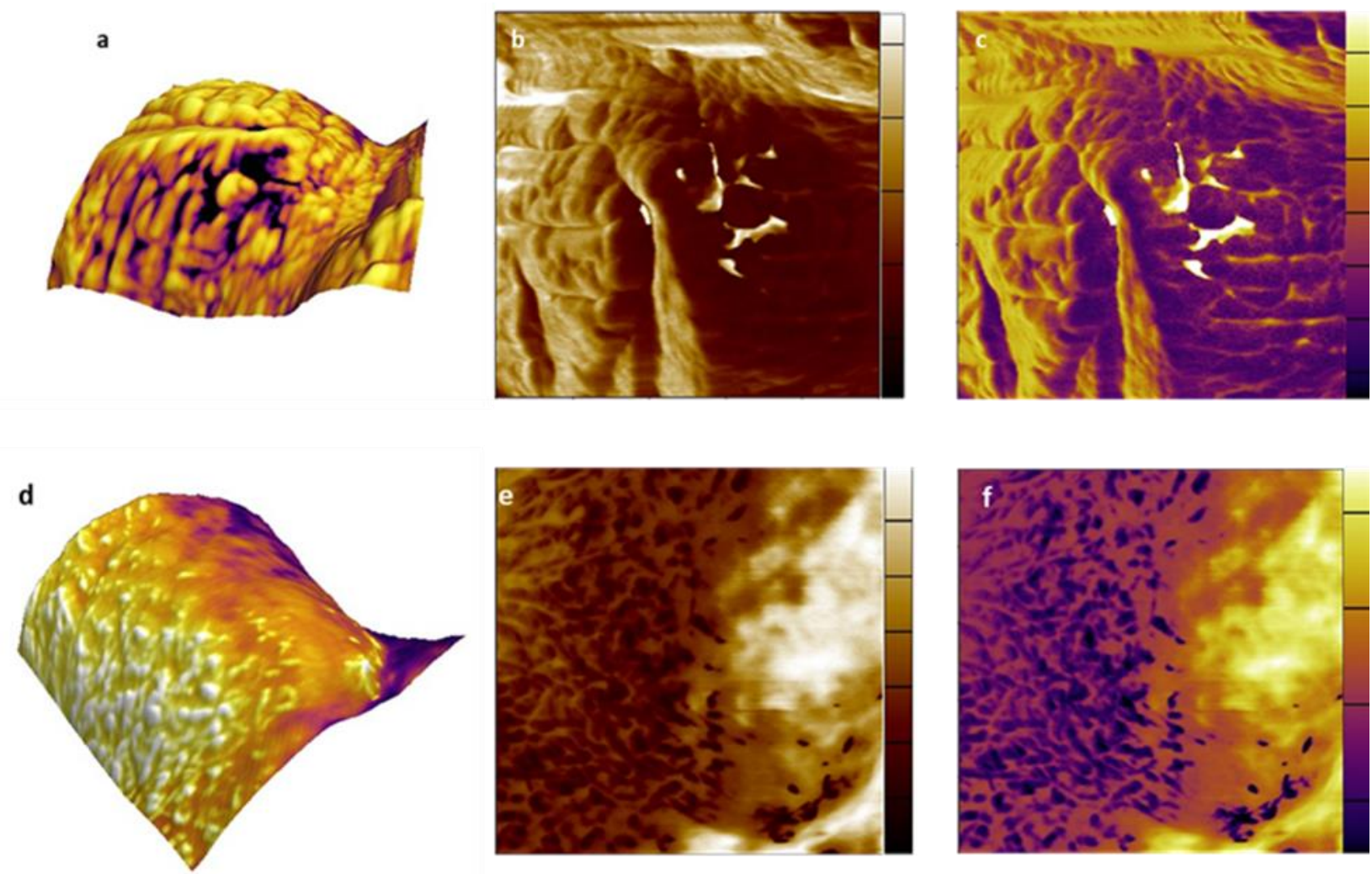

Figure 3. AFM imaging of sample without and with CNF. (a) to (c) refers to the sample without NFC and (d) to (f) refers to the sample with CNF (a) and (d) AFM 3D topography imaging. (b) and (e) Second amplitude (A2) images of bimodal AFM, corresponding to the dynamics of the first excited eigenmode and (c) and (f) correspond to the second frequency (f2) accordingly. The dimension of all images is $1 \mu \mathrm{m}$. From phase and amplitude image is obvious the existence of an extra material (CNF).

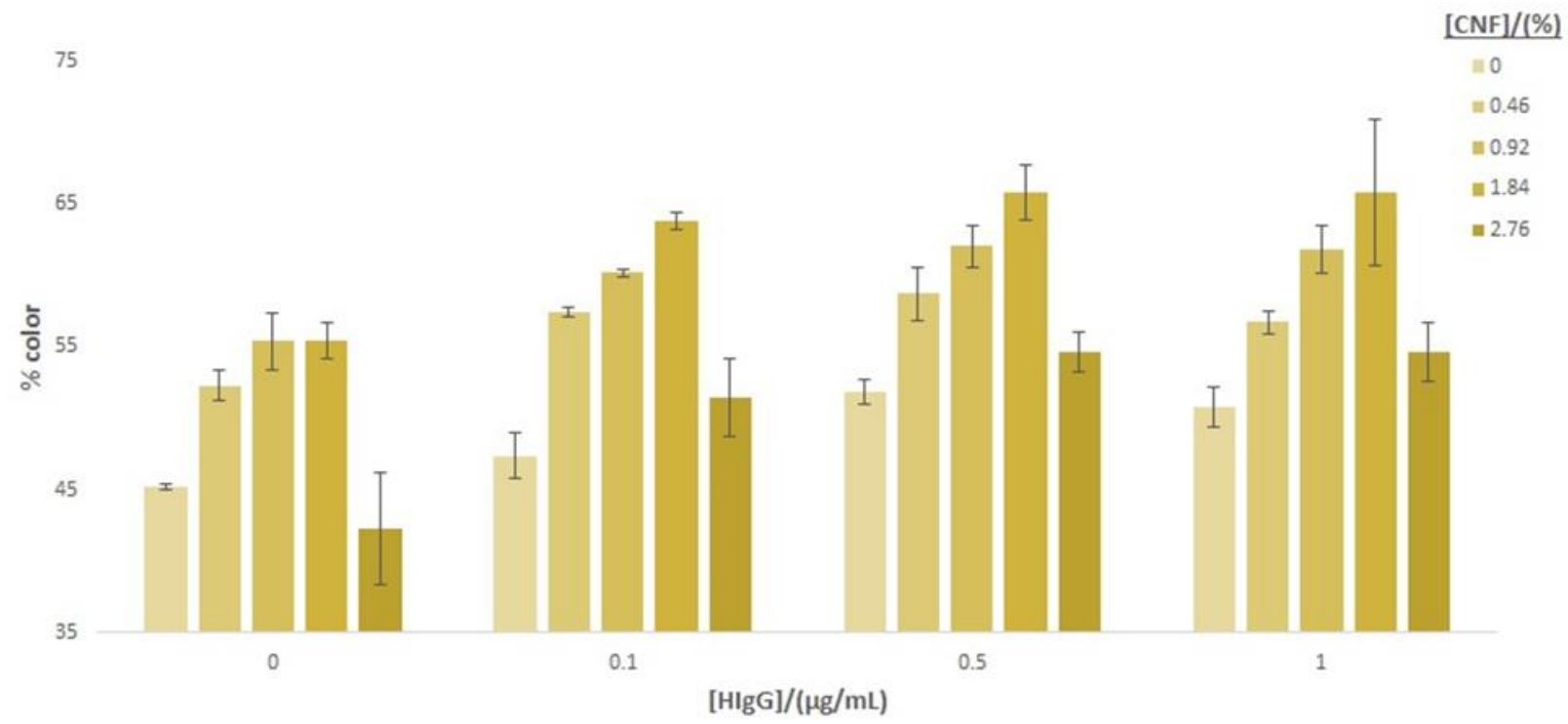

Figure 4. Comparison, at different concentrations of $\mathrm{HIgG}$, of the effect of adding different concentrations of CNF in the TL of LFs. 

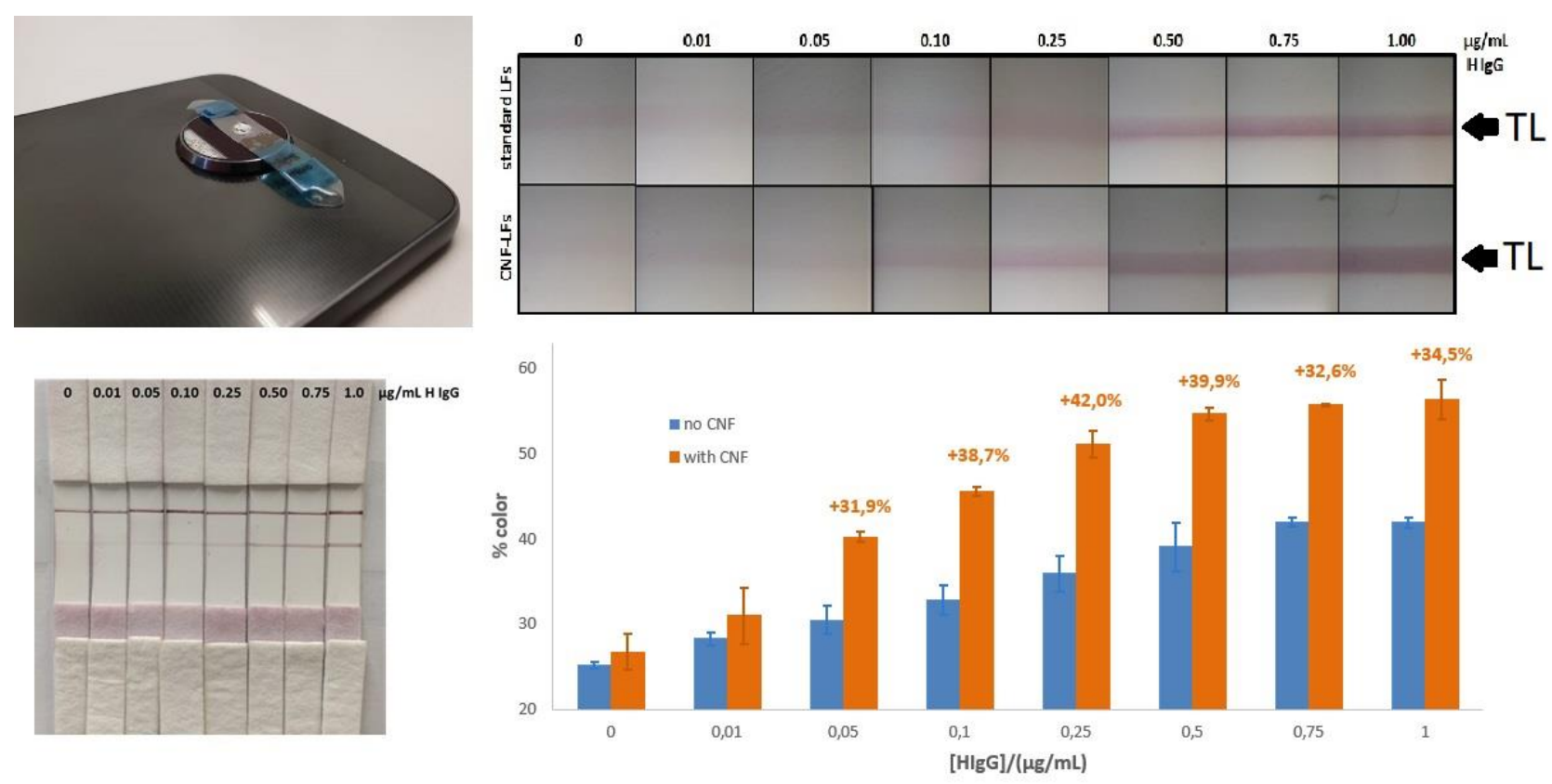

Figure 5. Data obtained from LFs assay performed with both standard and CNF-LFs and the images of the strips, also taken with Blips Micro Lens (inset).
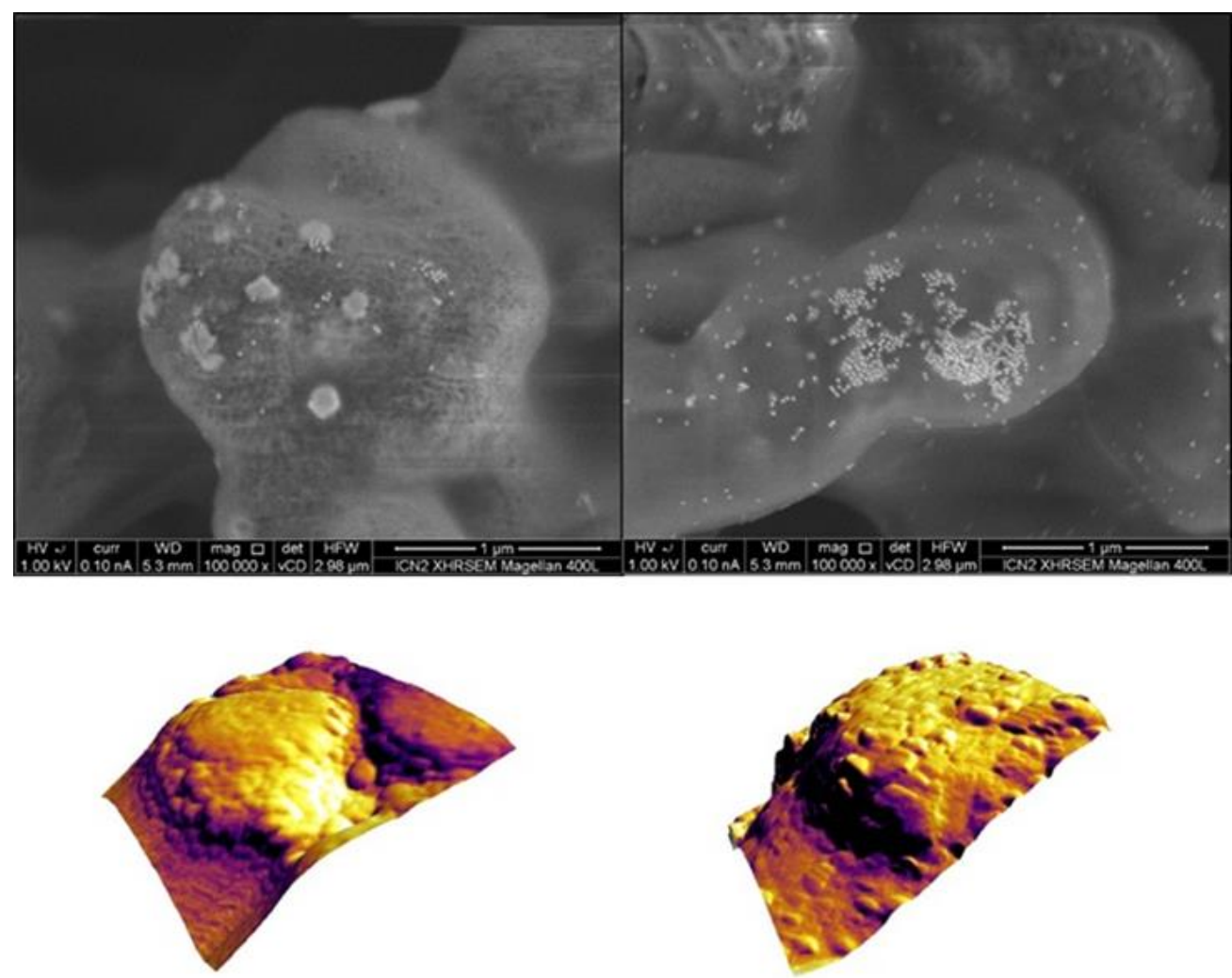

Figure 6. SEM (up) and AFM 3D topography (down) images of nitrocellulose corresponding to the TL of a standard LF (left) and of a CNF-LF (right). 